\title{
Cucurbitacin B as a Chinese Medicine Monomer Inhibits Cell Proliferation, Invasion, and Migration in Nasopharyngeal Carcinoma
}

\author{
Ning Xu, ${ }^{1}$ Bei-Bei Zhang, ${ }^{2}$ Meng-Zhe Yang, ${ }^{1}$ Xian-Yu Bai, ${ }^{1}$ Zhen-Qiang Liang, ${ }^{3}$ \\ Nan-Nan Cheng, ${ }^{1}$ An-Qiao Lv, ${ }^{1}$ Jian-Yu Yang, ${ }^{1}$ Xing-Zhe Guo, ${ }^{1}$ Ai-Jun Jiao, ${ }^{4}$ \\ and Yuan-Jiao Huang $\mathbb{D}^{5,6}$ \\ ${ }^{1}$ Graduate School of Guangxi Medical University, Nanning, China \\ ${ }^{2}$ Institute of Biomedical Research, Yunnan University, Kunming, China \\ ${ }^{3}$ Department of Otolaryngology-Head and Neck Surgery, The First Affiliated Hospital of Guangxi Medical University, \\ Nanning, China \\ ${ }^{4}$ Pharmaceutical College, Guangxi Medical University, Nanning, China \\ ${ }^{5}$ Life Science Institute, Guangxi Medical University, Nanning, China \\ ${ }^{6}$ School of Basic Medical Sciences, Guangxi Medical University, Nanning, China
}

Correspondence should be addressed to Yuan-Jiao Huang; hyjgxmu@126.com

Received 22 January 2021; Revised 19 February 2021; Accepted 23 February 2021; Published 12 March 2021

Academic Editor: Songwen Tan

Copyright (๑) 2021 Ning Xu et al. This is an open access article distributed under the Creative Commons Attribution License, which permits unrestricted use, distribution, and reproduction in any medium, provided the original work is properly cited.

\begin{abstract}
Nasopharyngeal carcinoma (NPC) is a malignant epithelial tumor in southern China. Cucurbitacin B (CuB) is a tetracyclic triterpene compound isolated from Cucurbitaceae plants which has anti-inflammation and antitumor properties and low toxic side effects. In this study, we use a series of wet experiments and network pharmacology analyses to explore the effects of CuB on cell proliferation, migration, invasion, and apoptosis of highly metastatic 5-8F NPC cells. The findings suggest that CuB inhibits NPC cells in a time- and dose-dependent manner and that cancer migration and invasion abilities decrease significantly after $\mathrm{CuB}$ treatments. Mechanistically, $\mathrm{CuB}$ could increase the proportion of cells in the G2/M phase and reduce it in the G0/G1 phase, leading to apoptosis. The network pharmacological analyses and wet experiments uncovered that the MAPK pathway is a central target by pathway enrichment analysis, affecting the fate of cancer cells and influencing proliferation and apoptosis. Taken together, our study reveals that $\mathrm{CuB}$ could effectively inhibit 5-8F NPC cell proliferation, migration, and invasion via cell cycle blockage and cell apoptosis. Collectively, we have shown that $\mathrm{CuB}$ is a promising anti-NPC candidate compound for future preclinical study.
\end{abstract}

\section{Introduction}

Nasopharyngeal carcinoma (NPC) is the most common malignant tumor of the nasopharynx. Although global NPC incidence is less than $1 / 100,000$, there are significant regional variations and a much higher incidence rate in North Africa and Southeast Asia, especially in the southern provinces of China. In 2018, there were 129,079 new NPC cases and 72,987 deaths around the world. Of these, more than $45 \%$ of the new cases were in China, several times higher than the global average incidence rate, and in some provinces, the rate exceeded $30 / 100,000[1,2]$. There are a number of pathological forms including the undifferentiated state [3], the hidden onset, mostly lymph node metastasis, and distant metastasis [4-6]. With the development of intensitymodulated radiotherapy (IMRT) treatment, patients with early stage NPC (stage I) who underwent radiation therapy alone experienced a 5-year survival rate (OS) of over $90 \%$ $[7,8]$. High survival rates are also seen in patients with stage II cancer who underwent radiotherapy alone, but $10-15 \%$ of patients develop recurrence and distant metastasis [9]. Combined chemotherapy can improve patient survival rate and 
reduce the rate of recurrence and metastasis, but the toxicity and side effects of chemotherapy drugs seem to do more harm than good $[10,11]$. Therefore, there is an urgent need to seek safe and effective radiotherapy adjuvant drugs with low toxicity and side effects which will help to reduce the recurrence and distant metastasis.

A number of studies have shown that many active components in Chinese herbal medicine have antitumor effects and that they are economical and multitarget, have low toxicity and side effects and, as such, are a current focus of antitumor drug research. Cucurbitacin is one of the main bioactive compounds found in Cucurbitaceae, such as cucumber [12]. It has been extracted from a variety of plants, has a wide range of pharmacological activities [13], such as cytotoxic, anti-inflammatory, and anticancer effects [14], and has been used as folk medicine for hundreds of years in countries such as India and China. Among the Cucurbitacin compounds, Cucurbitacin B $(\mathrm{CuB})$ is one of the most widely used active substances for in vivo and in vitro tumor inhibition studies. With low toxicity and side effects, it is a promising potential antitumor herbal drug [15]; however, no studies have been conducted on its effect on NPC.

In this study, we examined the effect of $\mathrm{CuB}$ on the highly metastatic NPC 5-8F cells and reveal its possible mechanism of action using in vitro wet experiments and bioinformatics analyses. Our findings suggest that $\mathrm{CuB}$ could effectively inhibit $5-8 \mathrm{~F}$ cell proliferation, migration, and invasion via cell cycle blockage and apoptosis. The network pharmacological analyses uncover that the MAPK pathway is a central target. Our findings show that $\mathrm{CuB}$ is a promising antiNPC candidate compound and should be the focus of future research.

\section{Material and Methods}

2.1. Materials. $5-8 \mathrm{~F}$ cells were purchased from Shanghai Institute of Cell Research, Chinese Academy of Sciences. $\mathrm{CuB}$ was purchased from MCE (United States). 1640 medium and FBS fetal bovine serum were purchased from Gibco (USA), Trypsin solution and DMSO were purchased from Amresco (USA), and BCA Protein Content Assay Kit and AnnexinV-FITC Apoptosis Assay Kit were purchased from Nanjing Kaiji Biotechnology Co., Ltd. CCK-8 reagent was purchased from Dojindo (Japan).

2.2. Cell Culture. NPC 5-8F cells were cultured in RPM-1640 containing $10 \%$ fetal bovine serum at $37^{\circ} \mathrm{C}$ and $5 \%$ carbon dioxide. The medium was changed daily, and the cells were isolated with $0.25 \%$ trypsin.

2.3. CCK-8 Assay. Cells were seeded into 96-well plates and cultured for 24,48 , and $72 \mathrm{~h}$. Cytotoxicity was detected by the CCK method. Absorbance at $450 \mathrm{~nm}$ was measured by an automatic enzyme plate reader (Bio-Tek, VT, USA). Cell mortality was calculated according to the following formula: inhibition rate $(\%)=($ average A450 in the control group - average A450 in the experimental group)/(average A450 in the control group - average A450 in the blank group) $\times 100 \%$. Each experiment was performed in triplicate.
2.4. Transwell Migration and Invasion Assay. $5-8 \mathrm{~F}$ cells in each group were resuspended in serum-free medium and a $2 \times 10^{5}$ cells $/ \mathrm{mL}$ cell suspension was prepared. A Transwell chamber was inoculated with $200 \mu \mathrm{L}$ cell suspension in the upper chamber and $600 \mu \mathrm{L}$ serum-free medium in the lower chamber. The cells were incubated at $37^{\circ} \mathrm{C}$ for $24 \mathrm{~h}$, and after which, sterile cotton swabs were used to remove the upper 5$8 \mathrm{~F}$ cells and were thoroughly washed in PBS. The cells were fixed in $4 \%$ paraformaldehyde for $30 \mathrm{~min}$ and stained in $0.1 \%$ crystal violet for $10 \mathrm{~min}$. Three fields were randomly selected under the microscope for photography, and the number of stained cells in crystal violet was calculated as the number of migrated cells. For the invasion assay, RPMI 1640 medium was added at a ratio of 1:5 to Matrigel, and the upper chamber of Transwell was coated. After drying, the same protocol as the cell migration assay above was performed, and the number of invading cells was counted.

2.5. Flow Cytometry Analysis of Apoptosis. The Annexin VFITC double-staining method was used to detect the effect of $\mathrm{CuB}$ on $5-8 \mathrm{~F}$ cell apoptosis. $1 \times 10^{6}$ cells were seeded into each well of a six-well plate, and different $\mathrm{CuB}$ concentrations $(0,200,400$, and $800 \mathrm{nM})$ were added after the cells adhered to the wall. Cells were incubated for $48 \mathrm{~h}$, then harvested, resuspended in cold PBS, and stained with the AnnexinFITC apoptosis detection kit according to the manufacturers' instructions. Cell apoptosis was then analyzed by flow cytometry. The experiment was performed in triplicate.

2.6. Flow Cytometry Analysis of Cell Cycle. 5-8F cells in the logarithmic growth stage were seeded into 6-well plates, with a total volume of each well of $2 \mathrm{~mL}$ and cell density of $1 \times 10^{6}$ cells/L. After the cells were treated with $\mathrm{CuB}$ at final concentrations of $0,200,400$, and $800 \mathrm{nM}$ for $48 \mathrm{~h}$, they were collected, rinsed twice with $0.01 \mathrm{~mol} / \mathrm{L} \mathrm{PBS}(\mathrm{pH}=7.2)$ for precooling, and fixed overnight with $75 \%$ cold ethanol at $4^{\circ} \mathrm{C}$. Before detection, the fixation solution was removed and propidium iodide (PI) was added and the samples stained at $4^{\circ} \mathrm{C}$ for $30 \mathrm{~min} .1 \times 10^{4}$ cells from each group were collected for flow cytometry detection. The MFLT SAAINC software was used to analyze the cell cycle phase ratio and early cell apoptosis rate. The experiment was performed in triplicate.

2.7. Bioinformatics Analysis. The PubChem database (https:// pubchem.ncbi.nlm.nih.gov) was used to find the $3 \mathrm{D}$ compound structure of $\mathrm{CuB}$, the PharmMapper database (http://www lilab-ecust.cn/pharmmapper) was used to obtain $\mathrm{CuB}$ targets, and the GeneCards (http://www.genecards.org) database was used to retrieve target NPC-related diseases. The online tools from (http://bioinformatics.psb.ugent.be/webtools/Venn) were used to make maps of NPC and CuB targets. The PPI analysis of protein interactions was performed using String (https:// string-db.org/cgi). The Cytoscape 3.6.1 software was used to construct the CUB-nasopharyngeal carcinoma target network map, and cytoHubba's MCC method was used to search for core genes. The David web site (https://david.ncifcrf.gov/) was used for GO enrichment analysis on the common targets and the Kobas online database (http://kobas.cbi.pku.edu.cn/ kobas3) was used for KEGG pathway enrichment analysis. 
2.8. Western Blot. Total protein $(40 \mu \mathrm{g})$ generated by cell lysis was separated on a $12 \%$ acrylamide gel $(80 \mathrm{~V}, 30 \mathrm{~min} ; 120 \mathrm{~V}$, $60 \mathrm{~min})$, then transferred to PVDF membrane $(270 \mathrm{~mA}$, $65 \mathrm{~min}$ ), blocked with $5 \%$ skim milk for $1 \mathrm{~h}$, washed three times with $0.1 \%$ TBST, and incubated in the primary antibody overnight at $4^{\circ} \mathrm{C}$. The membrane was then washed three times with TBST, $5 \mathrm{~min}$ each, and then incubated with diluted fluorescent secondary goat anti-rabbit antibody for $1 \mathrm{~h}$. The membrane was washed three times in TBST, $5 \mathrm{~min}$ each, and the Odyssey infrared fluorescence film scanner was used to sweep the film, adjust the required parameters, and save the picture. The antibodies used in this study were GADPH (Wuhan ProteinTech Company (China)), P-ERK and ERK (ABCAM (USA)), and BCL2 and BAX (Cell Signaling Technology (USA)). All the images were analyzed with ImageJ to calculate the gray value of the relevant target proteins.

2.9. Statistical Analysis. SPSS 25.0 was used for all statistical analysis. Measurement data was expressed as $x \pm s$. A $t$-test was used for the comparison of two sample means, and one-way analysis of variance was used for comparison of means between groups. A $P$ value $<0.05$ indicates statistical significance. For all the data presented in this study, * represents $P<0.05, * *$ represents $P<0.01$, and $* * *$ represents $P<0.001$. All experiments were repeated three times.

\section{Results}

3.1. CuB Inhibits NPC Proliferation. To explore the anticancer effects of $\mathrm{CuB}$, we cultured 5-8F NPC cells (a highly metastatic cell line). The CCK8 assay results showed that $\mathrm{CuB}$ had a concentration-time-dependent effect on NPC 5-8F cell proliferation (Figure 1). In detail, the inhibition rate of cell proliferation was $9.25 \pm 2.58 \%, \quad 19.63 \pm 2.14 \%, \quad 27.18 \pm 2.30 \%$, $32.30 \pm 3.18 \%, 46.77 \pm 3.08 \%\left(\mathrm{IC}_{50} 1435.84 \pm 272.36 \mathrm{nM}\right)$ for $24 \mathrm{~h} \mathrm{CuB}$ treatment at $200 \mathrm{nM}, 400 \mathrm{nM}, 600 \mathrm{nM}, 800 \mathrm{nM}$, and $1000 \mathrm{nM}$, respectively. Similarly, the cell proliferation inhibition rate was $27.77 \pm 2.85 \%, 37.72 \pm 4.09 \%, 44.88 \pm 3.63 \%$, $54.61 \pm 4.89 \%$, and $65.45 \pm 3.57 \%\left(\mathrm{IC}_{50} 622.94 \pm 68.67 \mathrm{nM}\right)$ for $48 \mathrm{~h} \mathrm{CuB}$ treatment at $200 \mathrm{nM}, 400 \mathrm{nM}, 600 \mathrm{nM}, 800 \mathrm{nM}$, and $1000 \mathrm{nM}$, respectively. Accordingly, the cell proliferation inhibition rate was $41.64 \pm 3.79 \%, 53.14 \pm 2.70 \%, 66.74 \pm$ $2.70 \%, 74.33 \pm 2.34 \%$, and $86.80 \pm 3.44 \%\left(\mathrm{IC}_{50}\right.$ was $314.79 \pm$ $30.48 \mathrm{nM})$ for $72 \mathrm{~h} \mathrm{CuB}$ treatment at $200 \mathrm{nM}, 400 \mathrm{nM}$, $600 \mathrm{nM}, 800 \mathrm{nM}$, and $1000 \mathrm{nM}$, respectively. Collectively, CuB could obviously inhibit cell proliferation in the highly metastatic cell line model.

3.2. CuB Suppresses Cell Migration and Invasion in NPC. Since $5-8 \mathrm{~F}$ is a highly metastatic cell line and metastasis is a clinical concern that needs to be addressed, we examined whether $\mathrm{CuB}$ has some effects on cell abilities such as migration and invasion. The results of the scratch experiment (Figure 2) showed that the migration rates of $5-8 \mathrm{~F}$ cells were $12.84 \pm 2.37 \%, 10.12 \pm 1.75 \%$, and $5.56 \pm 0.88 \%$ at $24 \mathrm{~h}$ and $21.45 \pm 2.13 \%, 12.29 \pm 2.11 \%$, and $10.43 \pm 0.93 \%$ at $48 \mathrm{~h}$ after treatment with $\mathrm{CuB}$ at 50,100, and $200 \mathrm{nM}$, respectively. Compared to the control group $(24 \mathrm{~h}, 18.98 \pm 0.97 \%$;

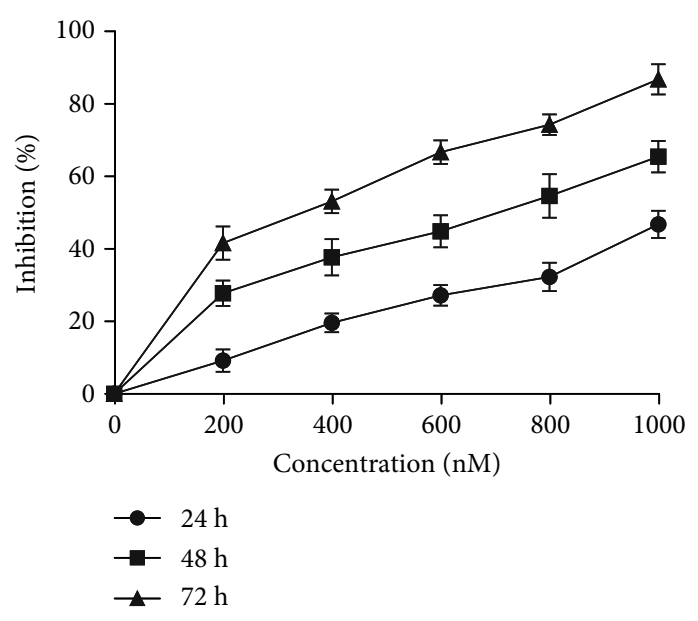

Figure 1: Effect of CuB on NPC cell proliferation.

$48 \mathrm{~h}, 35.15 \pm 5.21 \%), 50 \mathrm{nM} \mathrm{CuB}$ could significantly inhibit NPC $5-8$ F cells migration $(P<0.05)$, and the inhibition of $5-8 \mathrm{~F}$ migration was positively correlated with concentration.

To further validate these observations, Transwell migration experiments were performed. The results showed that the number of transmembrane cells in control and CuB treatment groups $(50 \mathrm{nM}, 100 \mathrm{nM}$, and $200 \mathrm{nM})$ was $386 \pm 7$, $187 \pm 9,142 \pm 2$, and $105 \pm 10$, respectively. Similarly, the Transwell-invasion experiment showed that the number of transmembrane cells in the control group and the CuB treatment groups $(50 \mathrm{nM}, 100 \mathrm{nM}$, and $200 \mathrm{nM})$ was $305 \pm 26$, $155 \pm 4,115 \pm 9$, and $29 \pm 14$, respectively. These results showed that as the $\mathrm{CuB}$ concentration increased, the migration and invasion ability of $5-8 \mathrm{~F}$ cells decreased significantly $(P<0.05)$ (Figure 3$)$. These results showed that $\mathrm{CuB}$ had some effect on metastatic abilities, such as migration and invasion, in NPC.

3.3. CuB Induces Cell Apoptosis in NPC. After seeing that cell proliferation, migration, and invasion were all significantly inhibited, we next explored whether $\mathrm{CuB}$ could affect NPC cell fate. Flow cytometry showed that after $48 \mathrm{~h}$ treatment with $\mathrm{CuB}$ at 200, 400, and $800 \mathrm{nM}$, the apoptosis rates were $15.58 \pm 3.09 \%, 24.6 \pm 1.83 \%$, and $31.76 \pm 5.06 \%$, respectively. Compared with the control group $(6.25 \pm 1.42 \%)$, the apoptosis rate was significantly increased and was correlated with concentration $(P<0.05)$ (Figure 4$)$. These data suggest that $\mathrm{CuB}$ could induce cell apoptosis in NPC and that this is closely related to drug concentration.

3.4. CuB Induces G2/M Phase Blockade in the Cell Cycle of NPC. We further examined the cell cycle to uncover the mechanism of action of CuB. Flow cytometry analysis of cell cycle showed that in $5-8 \mathrm{~F}$ treated with $\mathrm{CuB}$ at 200,400 , and $800 \mathrm{nM}$, the proportion of G2 phase cells increased $17.37 \pm$ $0.19 \%, 20.44 \pm 1.54 \%$, and $24.27 \pm 0.92 \%$, respectively, while the proportion of G0/G1 phase cells decreased, $57.58 \pm 1.19$ $\%, 53.16 \pm 0.42 \%$, and $49.63 \pm 2.13 \%$, respectively, compared with the control group (G2, $12.42 \pm 0.79 \%$, and $\mathrm{G} 0 / \mathrm{G} 1$, $60.51 \pm 2.13 \%$ ) (Figure 5). These results indicate that $\mathrm{CuB}$ could induce G2/M phase blockade in the NPC cell cycle. 

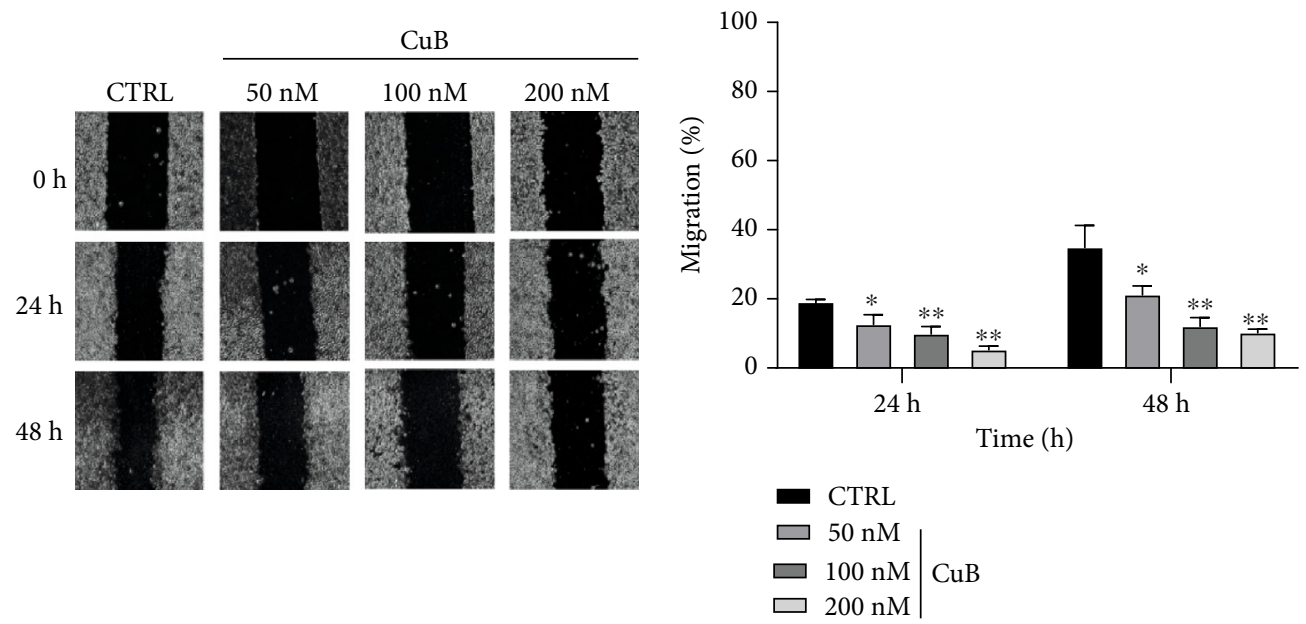

Figure 2: Effect of CuB on 5-8F cell migration.
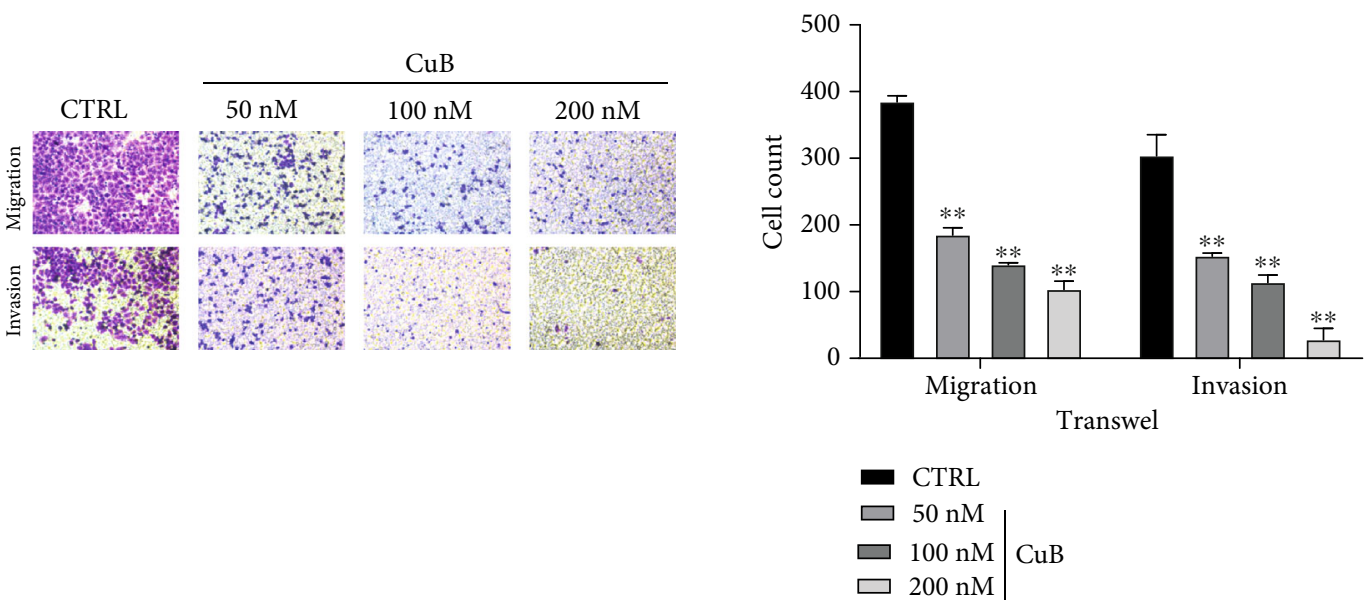

Figure 3: Effect of $\mathrm{CuB}$ on 5-8F cell migration and invasion.

3.5. Bioinformatics Analysis Uncovers MAPK as the Main Target of $\mathrm{CuB}$. To explore the overall anticancer effects of $\mathrm{CuB}$ against NPC, we performed bioinformatics analysis using a network pharmacology approach. In total, $357 \mathrm{CuB}$ action targets were obtained from the PharmMapper database and 1786 target genes from the GeneCards database. Among them, 116 common targets were validated (Figure 6(a)). The PPI analysis showed that MAPK1 was the most correlated gene among all the target genes, with a total of 30 interrelated genes (Figures 6(b) and 6(c)). MCC analysis of the common target genes with the Cytoscape plug-in showed that MAPK1 had the highest score (Figure $6(\mathrm{~d})$ ). Using $P<0.01$ as the threshold, 119 terms were obtained by GO enrichment analysis, of which, MAPK1 played a role in 28 terms and participated in three functions: BP, CC, and MF (Figure 6(e) shows the three functions of MAPK1 with the top 5 terms). The 28 functions in which MAPK1 is involved are shown in Figure 6(f). Using $P<0.01$ as the threshold, MAPK1 (ranked by $P$ value) was involved in 101 of the 159 pathways identified by the KEGG enrichment analysis. Figure 6(g) shows the MAPK1 position in the ranking by $P$ value of the top 15 pathways. MAPK1 mainly participates in biological processes through the MAPK pathway, and Figure 6(h) shows all the common target genes enriched in the MAPK pathway. Western blotting showed that the phosphorylation level of ERK (downstream of the MAPK pathway) decreased in a dose-dependent manner after $\mathrm{CuB}$ treatment (Figures 7(a) and 7(b)). After CuB treatment, levels of the proapoptotic protein Bax increased and of the apoptosis regulator Bcl-2 decreased (Figures 7(a) and 7(b)). Overall, the bioinformatics analysis and wet experiments show that MAPK is the central target of $\mathrm{CuB}$ against NPC.

\section{Discussion}

Previous studies have shown that there is no obvious toxicity to normal cells at $\mathrm{CuB}$ concentrations lower than $5-10 \mu \mathrm{M}$ [16]. Wakimoto et al. showed that when nude mice transplanted with MD-MB-231 tumor cells were injected with $\mathrm{CuB}(1 \mathrm{mg} / \mathrm{kg})$ intraperitoneally for 6 weeks, the tumor volume reduced by $55 \%$ compared to the control group while body weight did not reduce [17]. At present, no national food and drug administration has included $\mathrm{CuB}$ in the list of drugs or harmful substances [18]. The Australian Therapeutic Goods Administration has approved unrestricted use of 

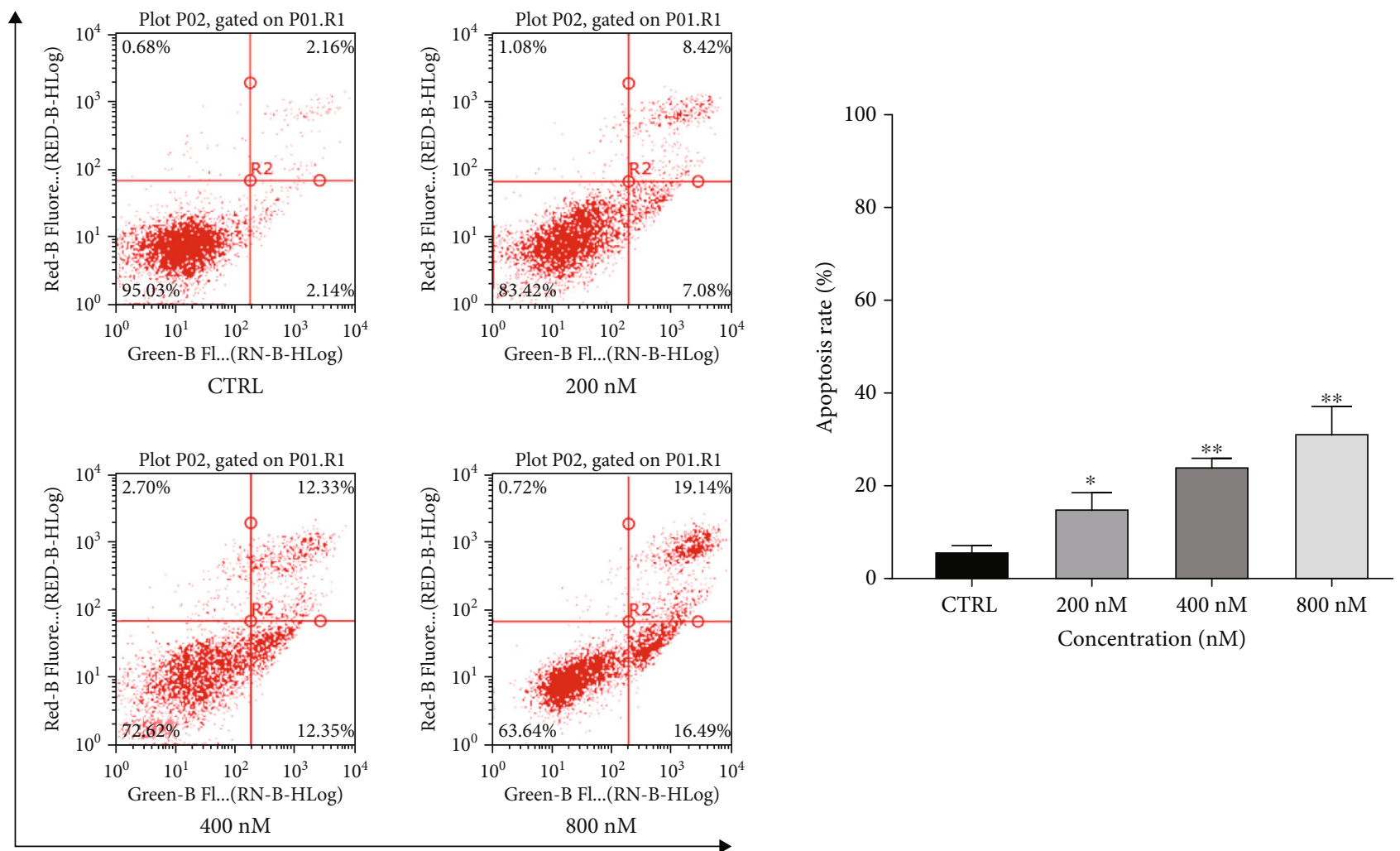

Annexin-V FITC

Figure 4: Effect of $\mathrm{CuB}$ on 5-8F cell apoptosis.


Figure 5: Effect of CuB on 5-8F cell cycle. 


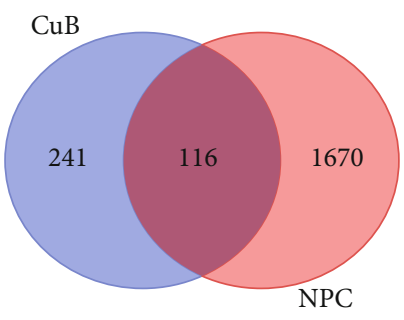

(a)

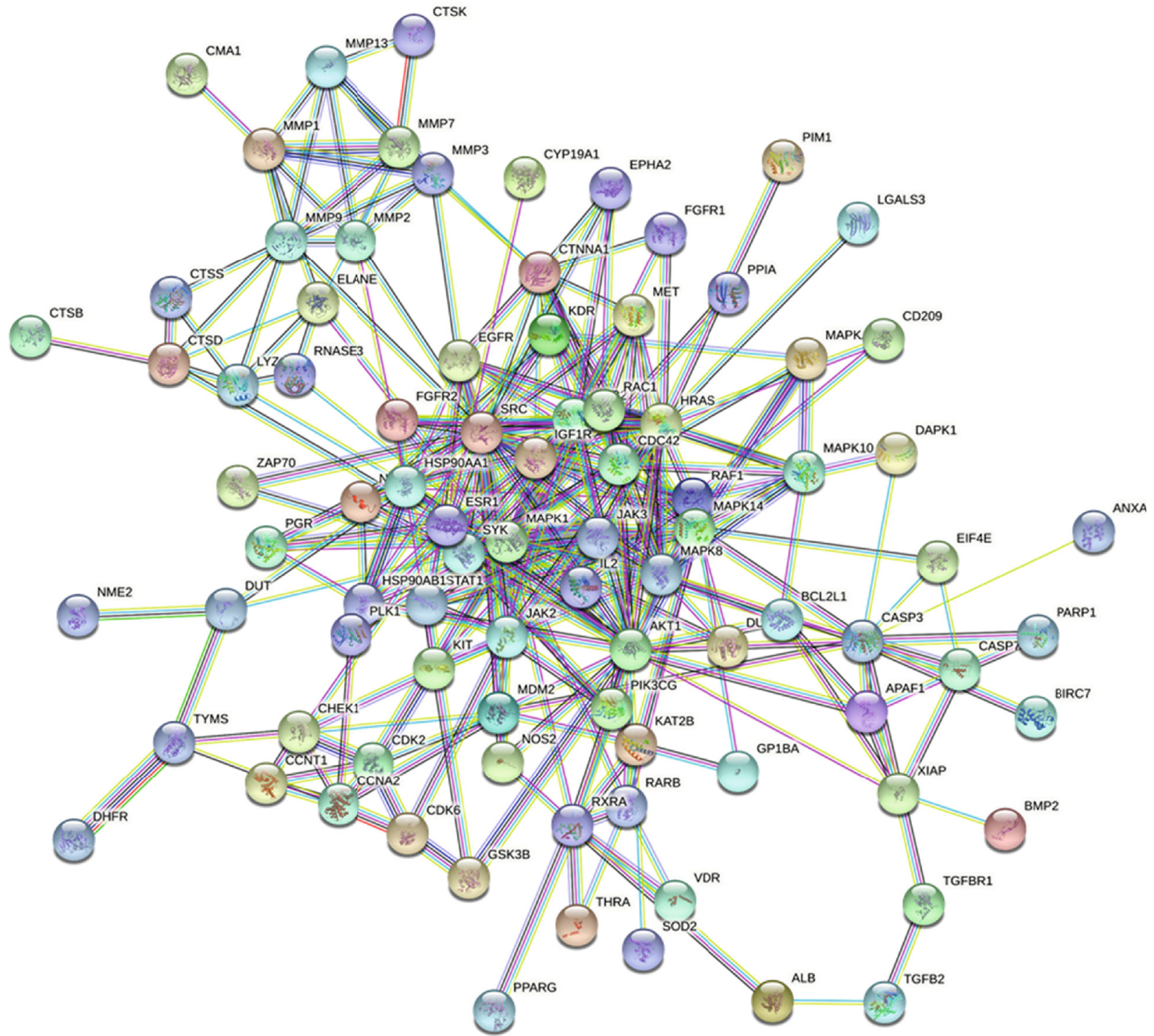

(b)

FIgUre 6: Continued. 


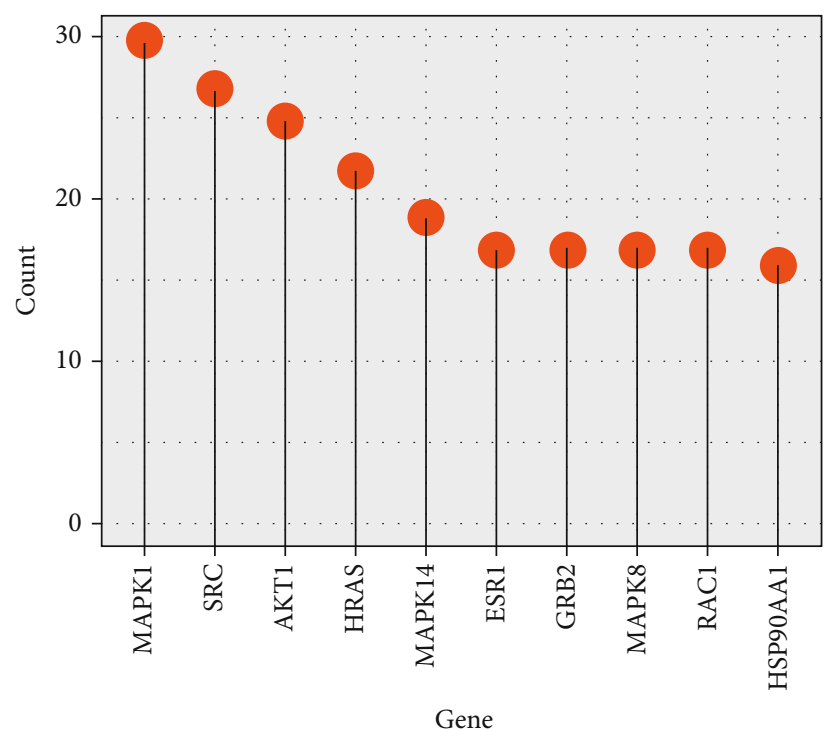

(c)
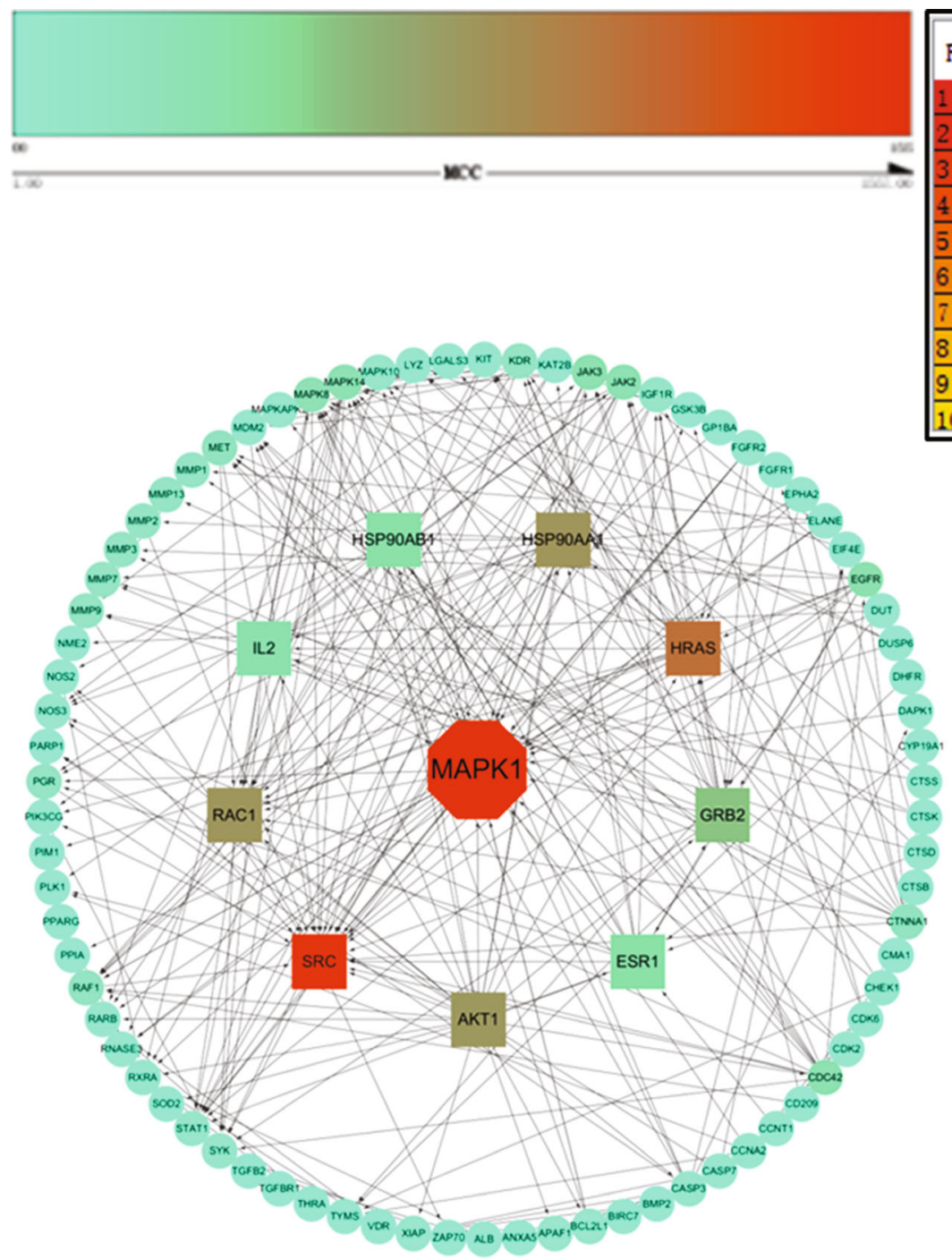

\begin{tabular}{|l|l|}
\hline Rank & $\begin{array}{l}\text { Hode } \\
\text { MaPK1 }\end{array}$ \\
\hline 1 & SRC \\
\hline 2 & HRAS \\
\hline 3 & HSP90AA1 \\
\hline 4 & RAC1 \\
\hline 5 & AKT1 \\
\hline 6 & GRB2 \\
\hline 7 & ESR1 \\
\hline 8 & HSP9OAB1 \\
\hline 9 & IL2 \\
\hline 10 & \\
\hline
\end{tabular}

(d)

Figure 6: Continued. 


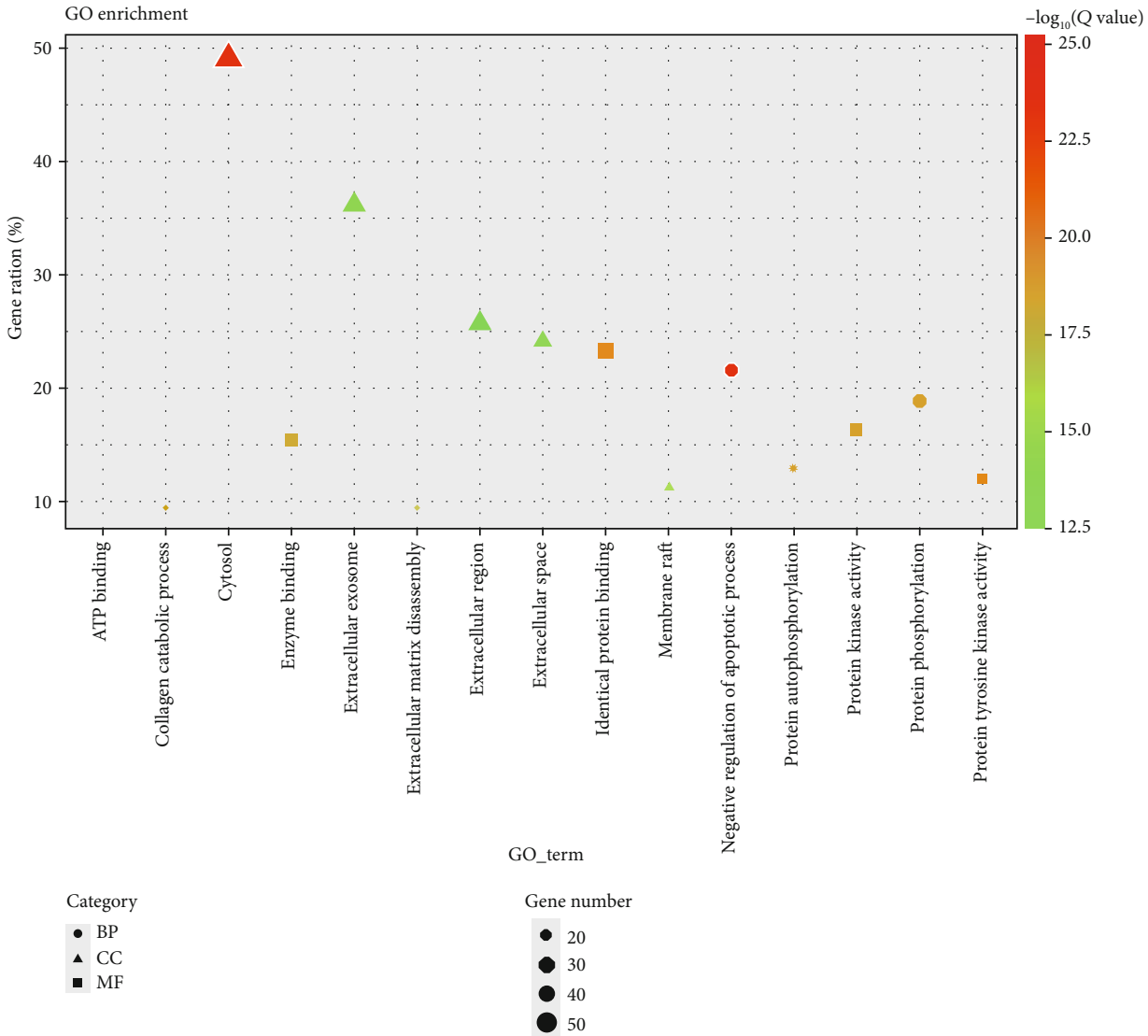

(e)

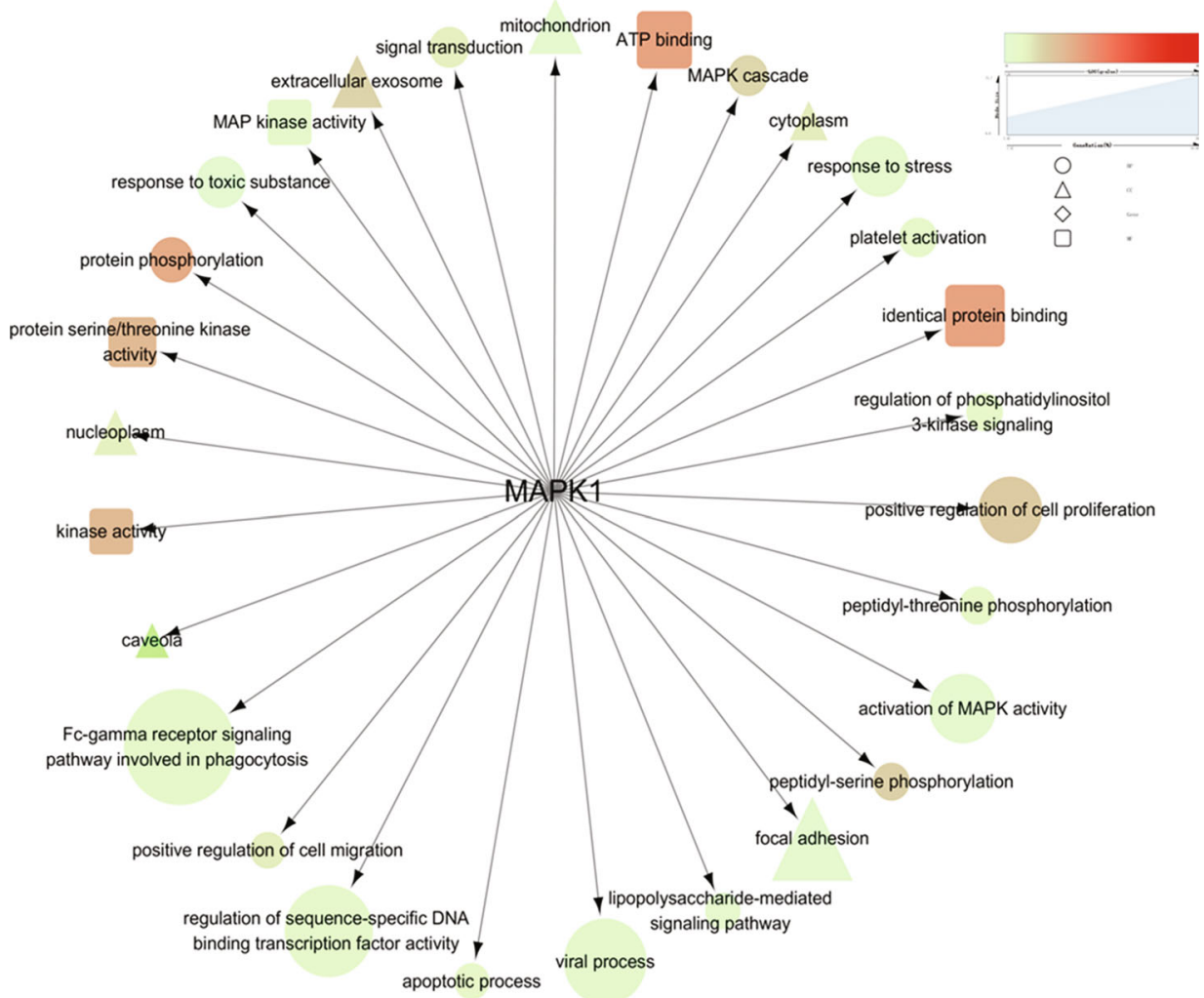

(f)

FIgURE 6: Continued. 


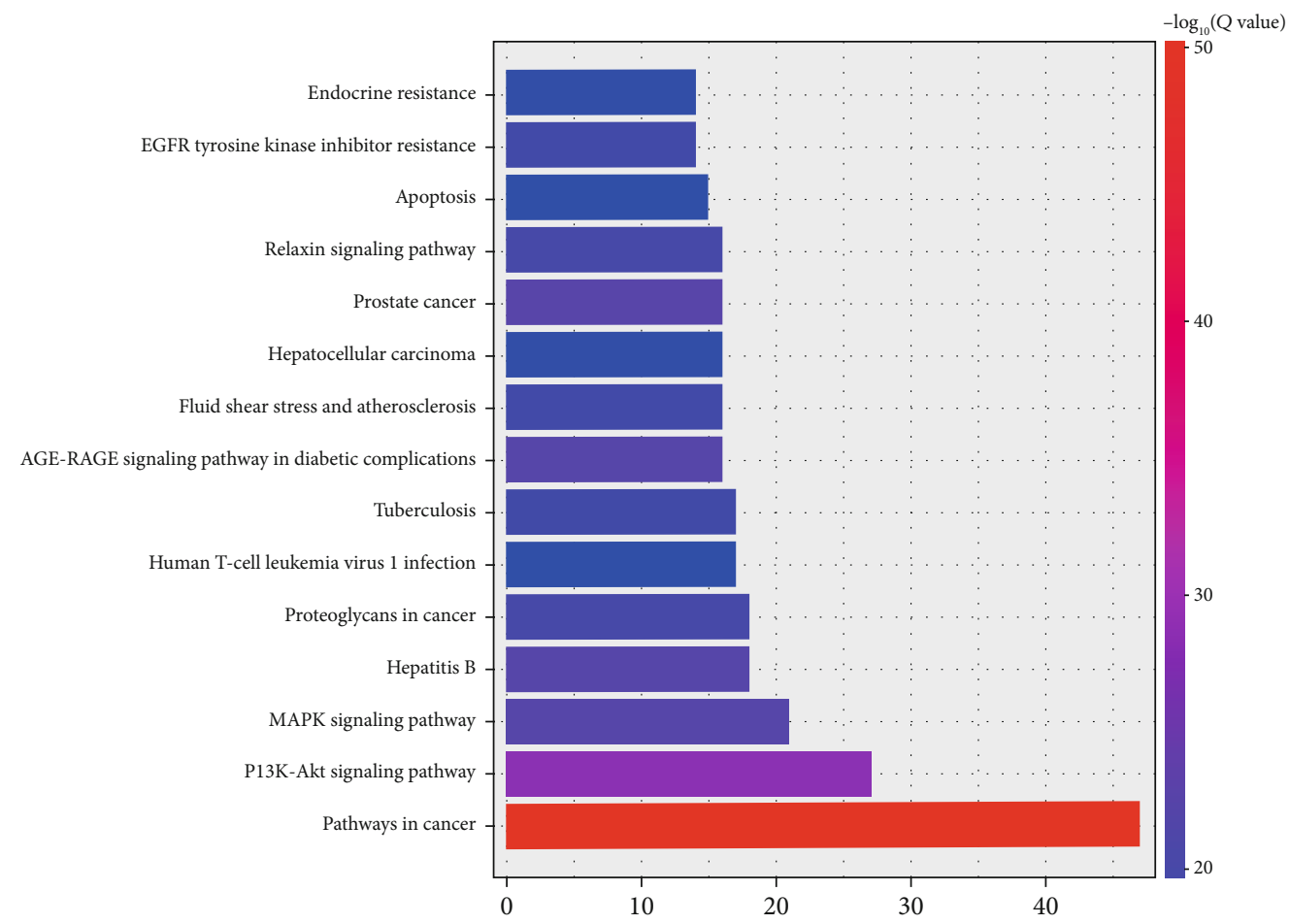

(g)

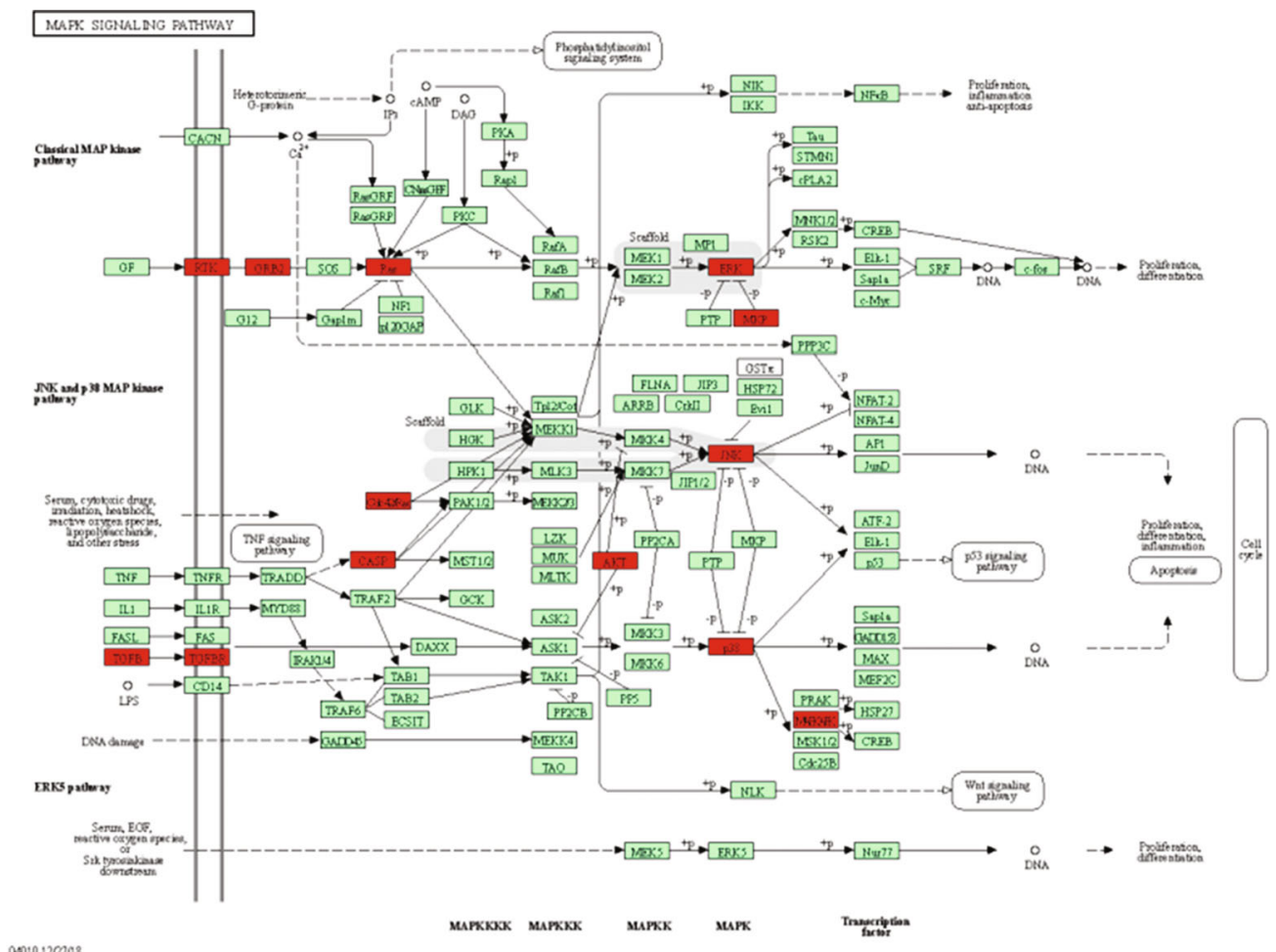

(h)

FIgURE 6: Bioinformatics analysis uncovers MAPK as the central target of CuB. (a) Venn diagram of NPC therapeutic targets in the GeneCards database and $\mathrm{CuB}$ therapeutic targets in the PharmMapper database. (b, c) PPI analysis showing that MAPK1 had the strongest interaction with the other proteins. (d) MCC calculation concluded that MAPK1 has the highest score and is the core gene of the common target genes. (e) GO enrichment analysis of the top 5 functional sets of BF, CC, and MF. (f) The functional set in which MAPK1 was involved as the core. (g) The KEGG analysis of the screened common target genes (top 15 pathway). (h) Genes enriched in the MAPK pathway. 

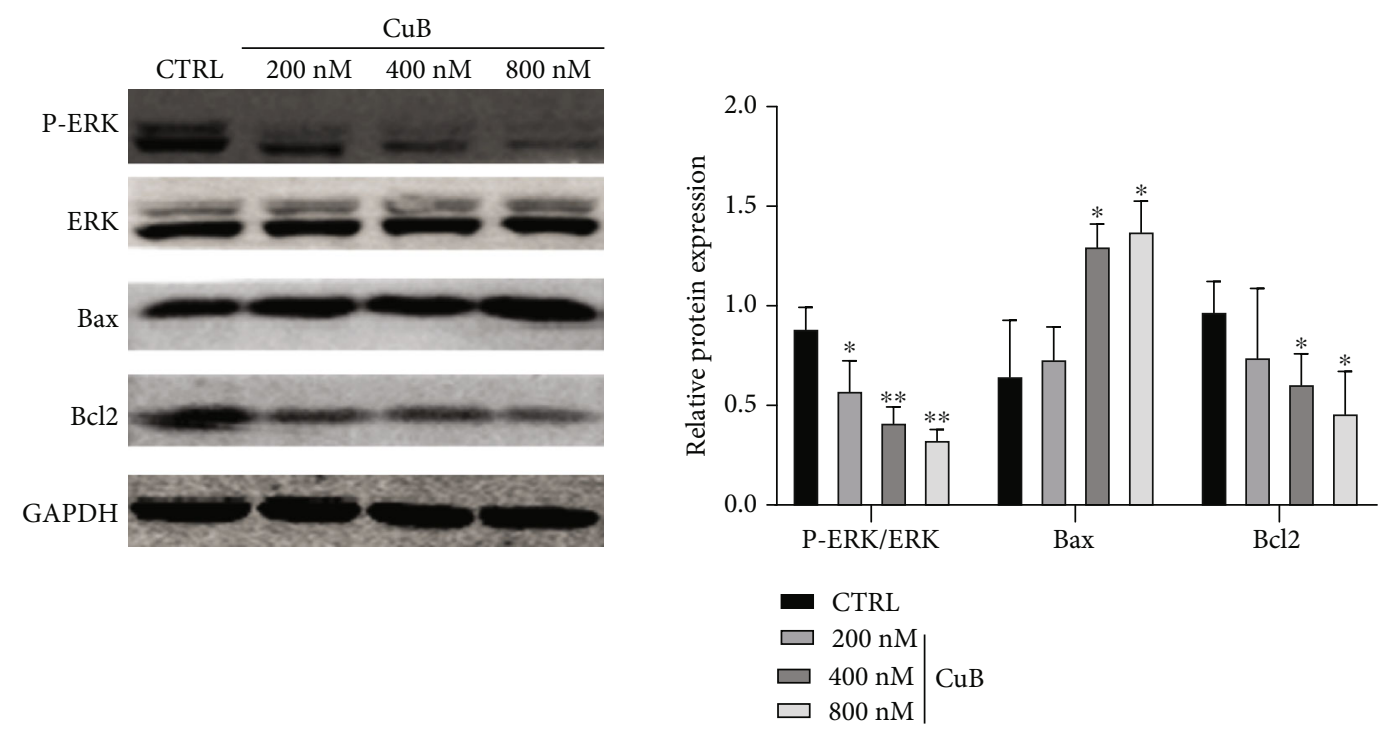

FIGURE 7: Relevant protein expression levels in 5-8F cells after $\mathrm{CuB}$ treatment.

$\mathrm{CuB}$ and encourages its use in combination with other drugs [19], and China has developed calabash tablets, 60 percent of the active ingredient is $\mathrm{CuB}$, which are used in the adjuvant treatment of hepatitis and primary liver cancer with the main side effect being mild gastrointestinal reactions. These studies confirm that $\mathrm{CuB}$ should be considered safe within therapeutic concentration ranges. In this study, when incubated with $5-8 \mathrm{~F}$ cells for 24,48 , and $72 \mathrm{~h}$, the IC50 of CuB was 1435.84 $\pm 272.36 \mathrm{nM}, 622.94 \pm 68.67 \mathrm{nM}$, and $314.79 \pm 30.48 \mathrm{nM}$, respectively. Overall, this information suggests that $\mathrm{CuB}$ has obvious anti-NPC potential at safe dosage levels.

Studies have shown that $\mathrm{CuB}$ can inhibit the invasion and migration of a variety of tumor cells, but the specific mechanism remains unclear. Promkan et al. found that $\mathrm{CuB}$ could effectively inhibit the invasion and migration of BRCA1 defective breast cancer cells [20], and Liang et al. showed that it could destroy the cytoskeleton of breast cancer cells, altering their biomechanical properties, and inhibiting their migration and invasion [21]. In non-small cell lung cancer, Garg et al. found that $\mathrm{CuB}$ inhibited cell invasion and migration by decreasing mortalin, hnRNP-K, vascular endothelial growth factor, matrix metalloproteinase 2, and fibronectin [22], and Piao et al. found that $\mathrm{CuB}$ could inhibit tumor invasion and migration by inhibiting tumor cell angiogenesis [23]. In this study, we showed that $\mathrm{CuB}$ could also inhibit invasion and migration of NPC $5-8 \mathrm{~F}$ cells, which is consistent with the results of previous studies.

Numerous studies have shown that $\mathrm{CuB}$ can inhibit the growth of many human cancer cells, including breast [24, 25], prostate [26], lung [27], ovarian [28], liver [29], pancreatic [30], larynx [31], kidney [32], and skin cancer [33]. Although many studies have demonstrated the anticancer activity of $\mathrm{CuB}$, the mechanism of its action is still unclear. It has been reported that $\mathrm{CuB}$ inhibits the growth of tumor cells and induces apoptosis by inhibiting the activation of signal transduction and transcriptional activator 3 (STAT3) and regulating its downstream genes such as cyclin D1, cyclin D2, and apoptosis-related genes like Bcl-2 and Bax [34, 35]. In this study, we found that MAPK1 is one of the most important targets of $\mathrm{CuB}$ through network pharmacology analysis.

Mitogen-activated protein kinases (MAPK) are the largest subfamily of serine/threonine protein kinases and are involved in many signal transduction pathways and play important roles in protein renewal, cell growth, transcription factor activation, chromatin modification, and gene expression. The MAPK pathway is a well-known stress sensing signal transduction pathway, which can respond to extraand intracellular stress signals and transmit environmental and self-derived signals into cells. Relevant studies have indicated that $\mathrm{CuB}$ is an inhibitor of JAK-STAT3, Wnt, PI3K/Akt, and MAPK signaling pathways, which play an important role in apoptosis and survival of cancer cells [36].

In human chronic myelogenous leukemia studies, $\mathrm{CuB}$ inhibited MAPK/ERK pathway activation in various human chronic myelogenous leukemia cells and inhibited STAT3 activation [37]. The analysis in this study showed that there was inhibition of MAPK/ERK activation, which led to the arrest of the G2/M cell cycle and apoptosis. Silva et al. reported that $\mathrm{CuB}$ can inhibit PI3 kinase and MAPK pathways, delay cell migration, and reduce cellular invasive potential [38]. It could also induce dose- and time-dependent apoptosis, inhibit MMP release and FAK activation, and downregulate Akt, ERK, and NF- $\kappa$ Bp65 phosphorylation. Zhang et al. found $\mathrm{CuB}$ had anticancer effects in the SH-SY5Y human neuroblastoma cell line [39]. CuB induced G2/M cell cycle arrest and apoptosis. This was accompanied by downregulation of CDK1 and cyclin B1, essential proteins in the cell cycle process. Another study has also reported that $\mathrm{CuB}$ inhibits proliferation and induces apoptosis of human osteosarcoma cells by regulating the JAK2/STAT3 and MAPK pathways [40]. In this study, MTT and Annexin V/propidium iodide staining showed that $\mathrm{CuB}(20-100 \mu \mathrm{M}$ dose) significantly reduced cell viability and induced apoptosis and inhibited cell migration. In conclusion, CuB's antitumor effect is mainly attributed to 
its induction of cell cycle arrest and apoptosis. The underlying mechanism of action may be attributed to the changes in key proteins involved in the regulation of cell cycle or apoptosis.

In conclusion, our study has shown that the Chinese medicine monomer $\mathrm{CuB}$ is a promising anticancer compound which affects cell proliferation, invasion, and migration in NPC cells. Mechanistically, its anticancer effects involve the cell cycle blockade and cell apoptosis, mediated through the MAPK signaling pathway. This shows that $\mathrm{CuB}$ is a promising agent for future NPC preclinical research and that this study also provides evidence that it might become part of a useful cancer therapy strategy.

\section{Data Availability}

The datasets used and/or analyzed during the current study are available from the corresponding author on the reasonable request.

\section{Conflicts of Interest}

The authors declare no conflict of interest.

\section{Authors' Contributions}

All authors made a significant contribution to the work reported, either in the conception, study design, execution, acquisition of data, analysis, or interpretation. Ning $\mathrm{Xu}$, Bei-Bei Zhang, and Meng-Zhe Yang contributed equally to this work.

\section{Acknowledgments}

This research was funded by the Project for Department of Science and Technology of Guangxi Zhuang Autonomous Region, China (grant number Guike AB19110052), the Natural Science Foundation of Guangxi, China (grant number 2015GXNSFAA139215), and the National Natural Science Foundation of China (grant number 81260405).

\section{References}

[1] F. Bray, J. Ferlay, I. Soerjomataram, R. L. Siegel, L. A. Torre, and A. Jemal, "Global cancer statistics 2018: GLOBOCAN estimates of incidence and mortality worldwide for 36 cancers in 185 countries," CA: a Cancer Journal for Clinicians, vol. 68, no. 6, pp. 394-424, 2018.

[2] J. Chen, J. Qi, B. Yu et al., "A retrospective study to compare five induction chemotherapy regimens prior to radiotherapy in the reduction of regional lymph node size in patients with nasopharyngeal carcinoma," Medical Science Monitor, vol. 24, pp. 2562-2568, 2018.

[3] M. L. K. Chua, J. T. S. Wee, E. P. Hui, and A. T. C. Chan, "Nasopharyngeal carcinoma," Lancet, vol. 387, no. 10022, pp. 1012-1024, 2016.

[4] Y. P. Mao, F. Y. Xie, L. Z. Liu et al., "Re-evaluation of 6th edition of AJCC staging system for nasopharyngeal carcinoma and proposed improvement based on magnetic resonance imaging," International Journal of Radiation Oncology • Biology • Physics, vol. 73, no. 5, pp. 1326-1334, 2009.
[5] M. Palazzi, E. Orlandi, P. Bossi et al., "Further improvement in outcomes of nasopharyngeal carcinoma with optimized radiotherapy and induction plus concomitant chemotherapy: an update of the Milan experience," International Journal of Radiation Oncology • Biology • Physics, vol. 74, no. 3, pp. 774-780, 2009.

[6] G.-Y. Liu, X. Lv, Y.-S. Wu et al., "Effect of induction chemotherapy with cisplatin, fluorouracil, with or without taxane on locoregionally advanced nasopharyngeal carcinoma: a retrospective, propensity score-matched analysis," Cancer Communications, vol. 38, no. 1, p. 21, 2018.

[7] A. W. M. Lee, W. M. Sze, J. S. K. Au et al., "Treatment results for nasopharyngeal carcinoma in the modern era: the Hong Kong experience," International Journal of Radiation Oncology - Biology • Physics, vol. 61, no. 4, pp. 1107-1116, 2005.

[8] N. Lee, J. Harris, A. S. Garden et al., "Intensity-modulated radiation therapy with or without chemotherapy for nasopharyngeal carcinoma: radiation therapy oncology group phase II trial 0225," Journal of Clinical Oncology, vol. 27, no. 22, pp. 3684-3690, 2009.

[9] J. Hu, Q. Huang, J. Gao et al., "Clinical outcomes of carbon-ion radiotherapy for patients with locoregionally recurrent nasopharyngeal carcinoma," Cancer, vol. 126, no. 23, pp. 5173$5183,2020$.

[10] F. Zhang, Y. Zhang, W. F. Li et al., "Efficacy of Concurrent Chemotherapy for Intermediate Risk NPC in the IntensityModulated Radiotherapy Era: a Propensity-Matched Analysis," Scientific Reports, vol. 5, no. 1, p. 17378, 2015.

[11] C. Xu, L. H. Zhang, Y. P. Chen et al., "Chemoradiotherapy versus radiotherapy alone in stage II nasopharyngeal carcinoma: a systemic review and meta-analysis of 2138 patients," Journal of Cancer, vol. 8, no. 2, pp. 287-297, 2017.

[12] P. K. Mukherjee, N. K. Nema, N. Maity, and B. K. Sarkar, "Phytochemical and therapeutic potential of cucumber," Fitoterapia, vol. 84, pp. 227-236, 2013.

[13] J. C. Chen, M. H. Chiu, R. L. Nie, G. A. Cordell, and S. X. Qiu, "Cucurbitacins and cucurbitane glycosides: structures and biological activities," Natural Product Reports, vol. 22, no. 3, pp. 386-399, 2005.

[14] M. A. Blaskovich, J. Sun, A. Cantor, J. Turkson, R. Jove, and S. M. Sebti, "Discovery of JSI-124 (cucurbitacin I), a selective janus kinase/signal transducer and activator of transcription 3 signaling pathway inhibitor with potent antitumor activity against human and murine cancer cells in mice," Cancer Research, vol. 63, no. 6, pp. 1270-1279, 2003.

[15] M. Clericuzio, M. Mella, P. Vita-Finzi, M. Zema, and G. Vidari, "Cucurbitane triterpenoids from Leucopaxillus gentianeus," Journal of Natural Products, vol. 67, no. 11, pp. 1823-1828, 2004.

[16] S. Garg, S. C. Kaul, and R. Wadhwa, "Anti-stress and glial differentiation effects of a novel combination of Cucurbitacin $\mathrm{B}$ and Withanone (CucWi-N): experimental evidence," Annals of Neurosciences, vol. 25, no. 4, pp. 201-209, 2019.

[17] N. Wakimoto, D. Yin, J. O'Kelly et al., "Cucurbitacin B has a potent antiproliferative effect on breast cancer cells in vitro and in vivo," Cancer Science, vol. 99, no. 9, pp. 1793-1797, 2008.

[18] S. Garg, S. C. Kaul, and R. Wadhwa, "Cucurbitacin B and cancer intervention: chemistry, biology and mechanisms (review)," International Journal of Oncology, vol. 52, no. 1, pp. 19-37, 2017.

[19] Therapeutic Goods Association, "Health Safety RegulationSubstances that may be used in listed medicines in Australia," Government of Australia, p. 86, 2011. 
[20] M. Promkan, S. Dakeng, S. Chakrabarty, O. Bogler, and P. Patmasiriwat, "The effectiveness of cucurbitacin B in BRCA1 defective breast cancer cells," PLoS One, vol. 8, no. 2, p. e55732, 2013.

[21] J. Liang, X. L. Zhang, J. W. Yuan et al., "Cucurbitacin B inhibits the migration and invasion of breast cancer cells by altering the biomechanical properties of cells," Phytotherapy Research, vol. 33, no. 3, pp. 618-630, 2019.

[22] S. Garg, H. Huifu, A. Kumari, D. Sundar, S. C. Kaul, and R. Wadhwa, "Induction of senescence in cancer cells by a novel combination of Cucurbitacin B and Withanone: molecular mechanism and therapeutic potential," The Journals of Gerontology. Series A, Biological Sciences and Medical Sciences, vol. 75, no. 6, pp. 1031-1041, 2020.

[23] X. M. Piao, F. Gao, J. X. Zhu et al., "Cucurbitacin B inhibits tumor angiogenesis by triggering the mitochondrial signaling pathway in endothelial cells," International Journal of Molecular Medicine, vol. 42, no. 2, pp. 1018-1025, 2018.

[24] Z. Q. Jin, J. Hao, X. Yang et al., "Higenamine enhances the antitumor effects of cucurbitacin B in breast cancer by inhibiting the interaction of AKT and CDK2," Oncology Reports, vol. 40, no. 4, pp. 2127-2136, 2018.

[25] S. Duangmano, P. Sae-lim, A. Suksamrarn, F. E. Domann, and P. Patmasiriwat, "Cucurbitacin B inhibits human breast cancer cell proliferation through disruption of microtubule polymerization and nucleophosmin/B23 translocation," BMC Complementary and Alternative Medicine, vol. 12, no. 1, p. 185, 2012.

[26] Y. Gao, M. S. Islam, J. Tian, V. W. Y. Lui, and D. Xiao, "Inactivation of ATP citrate lyase by Cucurbitacin B: a bioactive compound from cucumber, inhibits prostate cancer growth," Cancer Letters, vol. 349, no. 1, pp. 15-25, 2014.

[27] P. Lu, B. Yu, and J. Xu, "Cucurbitacin B regulates immature myeloid cell differentiation and enhances antitumor immunity in patients with lung cancer," Cancer Biotherapy and Radiopharmaceuticals, vol. 27, no. 8, pp. 495-503, 2012.

[28] Y. Qu, P. Cong, C. Lin, Y. Deng, J. Li-Ling, and M. Zhang, "Inhibition of paclitaxel resistance and apoptosis induction by cucurbitacin B in ovarian carcinoma cells," Oncology Letters, vol. 14, no. 1, pp. 145-152, 2017.

[29] K. T. Chan, F. Y. Meng, Q. Li et al., "Cucurbitacin B induces apoptosis and S phase cell cycle arrest in BEL-7402 human hepatocellular carcinoma cells and is effective via oral administration," Cancer Letters, vol. 294, no. 1, pp. 118-124, 2010.

[30] N. H. Thoennissen, G. B. Iwanski, N. B. Doan et al., "Cucurbitacin B induces apoptosis by inhibition of theJAK/STATPathway and potentiates antiproliferative effects of gemcitabine on pancreatic cancer cells," Cancer Research, vol. 69, no. 14, pp. 5876-5884, 2009.

[31] T. Liu, H. Peng, M. Zhang, Y. Deng, and Z. Wu, "Cucurbitacin $\mathrm{B}$, a small molecule inhibitor of the Stat3 signaling pathway, enhances the chemosensitivity of laryngeal squamous cell carcinoma cells to cisplatin," European Journal of Pharmacology, vol. 641, no. 1, pp. 15-22, 2010.

[32] C. J. Henrich, C. L. Thomas, A. D. Brooks et al., "Effects of cucurbitacins on cell morphology are associated with sensitization of renal carcinoma cells to TRAIL-induced apoptosis," Apoptosis, vol. 17, no. 1, pp. 79-89, 2012.

[33] T. Zhang, Y. Li, K. A. Park et al., "Cucurbitacin induces autophagy through mitochondrial ROS production which counteracts to limit caspase-dependent apoptosis," Autophagy, vol. 8, no. 4, pp. 559-576, 2014.
[34] N. Jing and D. J. Tweardy, "Targeting Stat3 in cancer therapy," Anti-Cancer Drugs, vol. 16, no. 6, pp. 601-607, 2005.

[35] J. Sun, M. A. Blaskovich, R. Jove, S. K. Livingston, D. Coppola, and S. M. Sebti, "Cucurbitacin Q: a selective STAT3 activation inhibitor with potent antitumor activity," Oncogene, vol. 24, no. 20, pp. 3236-3245, 2005.

[36] Y. Cai, X. Fang, C. He et al., "Cucurbitacins: a systematic review of the phytochemistry and anticancer activity," The American Journal of Chinese Medicine, vol. 43, no. 7, pp. 1331-1350, 2015.

[37] K. T. Chan, K. Li, S. L. Liu, K. H. Chu, M. Toh, and W. D. Xie, "Cucurbitacin B inhibits STAT3 and the Raf/MEK/ERK pathway in leukemia cell line K562," Cancer Letters, vol. 289, no. 1, pp. 46-52, 2010.

[38] I. T. Silva, F. C. Geller, L. Persich et al., "Cytotoxic effects of natural and semisynthetic cucurbitacins on lung cancer cell line A549," Investigational New Drugs, vol. 34, no. 2, pp. 139-148, 2016.

[39] Q. Zheng, Y. Liu, W. Liu et al., "Cucurbitacin B inhibits growth and induces apoptosis through the JAK2/STAT3 and MAPK pathways in SH-SY5Y human neuroblastoma cells," Molecular Medicine Reports, vol. 10, no. 1, pp. 89-94, 2014.

[40] Z. R. Zhang, M. X. Gao, and K. Yang, "Cucurbitacin B inhibits cell proliferation and induces apoptosis in human osteosarcoma cells via modulation of the JAK2/STAT3 and MAPK pathways," Experimental and Therapeutic Medicine, vol. 14, no. 1, pp. 805-812, 2017. 